\title{
ECOTROPHELIA 2017: Una oportunidad para el enfoque interdisciplinar en el aprendizaje basado en proyectos.
}

Esther González Aurignac ${ }^{\mathrm{b}}$, Purificación García Segovia a , Javier Martínez Monzóa, Gabriel García Martínez y María Jesús Pagán Moreno .

${ }^{\mathrm{a}}$ ETSIAMN, ${ }^{\mathrm{b}}$ ETSID

\begin{abstract}
The present educational improvement project based on a real professional framework and developed by teams of interdisciplinary students, demonstrates that the use of active learning methodologies stimulate students motivation and their capacity for continuous learning, preparing them for the demanding of the dynamic and changing future professional world. The main objective of this teaching innovation consisted in the design of an eco-innovative food product from its concept and image to the business plan and to present it at the ECOTROPHELIA 2017 competition. The teams participating in the project belonged to two different degrees, students of ETSID (Degree in Design Engineering and Product Development) and ETSIAMN (Degree in Food Science and Technology). The interdisciplinary teamwork developed by our UPV students, was the winner of the Ecotrophelia Spain 2017 contest and represented our country in the European contest in London in November 2017.
\end{abstract}

Keywords: interdisciplinarity, projects, creativity, teamwork, active learning, critical thinking and planning.

\section{Resumen}

El presente proyecto de innovación y mejora educativa basado en un marco real y desarrollado por equipos de alumnos interdisciplinares, demuestra que el uso de metodologías activas de aprendizaje, estimula la motivación del estudiante y su capacidad de aprendizaje continuo, preparándolo para las demandadas del exigente mundo profesional actual y futuro. El objetivo principal de la presente innovación docente consistió, en el diseño de productos alimentarios ecoinnovadores desde su concepto e imagen, hasta el plan de negocio, para su presentación posterior en el certamen ECOTROPHELIA 2017. Los equipos participantes en el proyecto pertenecían a dos titulaciones diferentes, alumnos de la ETSID (Grado en Ingenieria del Diseño y Desarrollo de Productos) y de la ETSIAMN (Grado en Ciencia y Tecnología de los Alimentos). El trabajo en equipo $e$ interdisciplinar desarrollado por estos alumnos de la UPV, fue el ganador del certamen Ecotrophelia España 2017 y representó a nuestro país en el certamen Europeo en Londres en noviembre 2017. 
ECOTROPHELIA 2017: Una oportunidad para el enfoque interdisciplinar en el aprendizaje basado en proyectos.

Palabras clave: interdisciplinaridad, proyectos, creatividad, trabajo en equipo, aprendizaje activo, pensamiento critico y planificación

\section{Introducción}

\subsection{Contextualización de la experiència.}

El proceso de convergencia al Espacio Europeo de Educación Superior establece la incorporación de metodologías activas de aprendizaje (Arias, 2003), por ello entendemos que debemos realizar una reflexión profunda sobre los procesos de enseñanza-aprendizaje tradicionales y pasivos, resaltando la urgencia de innovar en el desarrollo de vías de integración de los mismos, con las necesidades reales del mundo profesional. Cada vez más las empresas son organizaciones activas y dinámicas, basadas en la capacidad de aprendizaje continuo de su personal (Edvinsson, 1997). Por ello, consideramos de gran importancia, la formación de nuestros alumnos para adecuarse a las demandas del cambiante entorno empresarial al que deberán enfrentarse en un futuro cercano y que solicita profesionales “ con la capacidad de aprender a saber, aprender a aprender y aprender a trabajar juntos” (Delors, 1996).

El presente proyecto de innovación y mejora educativa, fue encaminado a buscar un puente entre la universidad y el mundo laboral (González-Aurignac,2013). Planteamos una innovación metodológica con la que construir un entorno que beneficiase el aprendizaje (Fulla, 2002) a través de la motivación de los alumnos ante una situación real de diseño y materialización de un producto dentro de un equipo interdisciplinar.

La implementación del proyecto en el desarrollo de productos alimentarios, se realizó entre dos colectivos de estudiantes procedentes de la ETSID y de la ETSIAMN. Estos colectivos trabajaron en colaboración para desarrollar el diseño de cuatro productos alimentarios y sus respectivas filosofías e imagenes de marca, envases y logotipos, así como también los planes de negocio correspondientes a cada producto.

- Los alumnos de Generación Espontanea de la ETSIAMN: Trabajarón en el diseño de un producto alimentario ecoinnovador para su presentación en el certamen ECOTROPHELIA 2017 y el plan de negocio asociado al mismo.

- Los alumnos de la asignatura de Color y diseño de la ETSID: Trabajarón sobre la filosofía e imagen de marca del producto incluyendo el diseño de los envases, logotipos e imagenes coorporativas de cada uno de los productos alimantarios propuestos por los compañeros de la ETSIAMN para ECOTROPHELIA 2017.

\subsection{Justificación y motivación}

Para contextualizar el arranque de esta colaboración, podemos remontarnos a cinco años atrás, coincidiendo con la implantación de los nuevos grados, cuando la unidad docente de Nutrición y Restauración Colectiva, del departamento de Tecnología de Alimentos, decidió implantar la creatividad y la flexibilidad ofrecida por herramientas propias del mundo del diseño, como es el Design Thinking (DT), para abordar los conocimientos de las 
asignaturas implicadas en su unidad docente y con el fin de trabajar también, las competencias transversales de las asignaturas.

De este modo, durante el semestre correspondiente los alumnos en equipos debían, manejando las herramientas básicas del DT, diseñar, prototipar y presentar un nuevo alimento, que supusiese una innovación en el mercado. Desde esta primera iniciativa , hemos ido enviando anualmente equipos de nuestros alumnos al concurso nacional Ecotrophelia, que además cuenta con una fase europea, donde va, representando a cada país, el equipo ganador en la fase nacional. En dos ocasiones (2011 y 2015) alumnos de la UPV han resultado vencedores en la fase española acudiendo así, a la europea.

Como consecuencia de estas primeras experiencias, nos dimos cuenta de la oportunidad del enfoque interdisciplinaridad y de la necesidad del trabajo en equipo entre alumnos procedentes de titulaciones complementarias, para alcanzar la profesionalidad que demanda la sociedad actual. Constatamos que, no sólo es importante presentar un producto innovador, sino que, aspectos como la filosofía e imagen del producto, el marketing y el business plan, son fundamentales para que las propuestas fuesen completas y especialmente, para poder competir en la fase europea. Por ello, vimos la urgencia de introducir en el proceso la interdisciplinaridad en el proyecto, como solución para obtener resultados completos, coherentes y mejorados. Gracias a las posibilidades que la UPV nos ofrece, al tener titulaciones que se complementan en la vida profesional, iniciamos este proyecto de innovación y mejora educativa entre las dos escuelas, que nos ha permitido trabajar de forma activa las competencias propias de cada asignatura y en paralelo también las transversales (conocimiento de los problemas del entorno, comunicación efectiva, trabajo en equipo, responsabilidad ética y medioambiental, planificación y gestión del tiempo,....) consiguiendo crear una oportunidad para alcanzar el " aprendizaje complejo, el cual requiere comprensión, aplicación, análisis y síntesis crítica de los conocimientos" (Lave,1991). Así, se ha pretendido fomentar la formación del estudiante en una serie de conocimientos vinculados a las asignaturas implicadas y relacionados con la situación real de sus profesiones futuras (Brown, 1998), consiguiendo así un aprendizaje profundo (Bain,2005).

\section{Objetivos}

El objetivo general de la presente experiencia de innovación y mejora educativa, fue desarrollar un proyecto de diseño y materialización de un producto innovador en una situación real, propia del futuro profesional de las titulaciones implicadas en el proceso. Para ello se planteó el proyecto con equipos de alumnos multidisciplinares. Dichos equipos tenían como objetivo final la presentación de un producto alimentario eco-innovador al certamen Ecotrophelia, teniendo el rol de responsables del desarrollo del alimento, los alumnos de la ETSIAMN y el rol de responsables del pakaging, logo e imagen de marca del producto alimentario, los alumnos de la ETSID. Para la consecución de dicho objetivo se plantearon los siguientes objetivos específicos: propiciar la cooperación y colaboración entre colectivos de alumnos de las titulaciones complementarias, fomentar la puesta en común de los conocimientos adquiridos durante el proceso de aprendizaje, facilitar la 
ECOTROPHELIA 2017: Una oportunidad para el enfoque interdisciplinar en el aprendizaje basado en proyectos.

sinergia de ideas y significados, aprender a contrastar tanto la información como los conocimientos, fomentar la toma de decisiones y la planificación del trabajo en equipo .

Además, y como objetivo complementario se pretendió determinar que competencias transversales estarían asociadas al desarrollo de este tipo de proyecto de innovación y mejora educativa en vistas a la implementación de las mismas en la presentación de Proyectos Fin de Grado Colaborativos u otro tipo de actividad similar.

\section{Desarrollo de la innovación}

\subsection{Implementación de los equipos interdisciplinares en el proceso de creación de productos alimentarios innovadores.}

Esta fase de implementación se realizó entre dos colectivos de alumnos: los alumnos de la generación espontanea pertenecientes al Grado en Ciencia y Tecnología de los alimentos de la ESTIAMN y los alumnos de la asignatura de Color y diseño del Grado en Ingenieria del Diseño y Desarrollo de Productos de la ETSID.

Los primeros idearon el producto alimentario y realizaron el plan de negocio, por otro lado los segundos fueron los encargados de crear y desarrollar la filosofía e imagen tanto del producto como de la empresa. En el caso de los alumnos de la ETSIAMN se trabajó con cuatro grupos de 5-6 alumnos, mientras que en lo referente a los alumnos de la ETSID se crearon 12 subgrupos de 3 alumnos. Se organizó la colaboración de tal forma, que cada tres grupos de la escuela de Diseño trabajaran sobre una misma propuesta de las cuatro realizadas por los equipos de Tecnología de Alimentos.

La finalidad del trabajo de los alumnos de la ETSIAMN era la creación de cuatro empresas alimentarias (startup) con un producto alimentario innovador estrella para cada una de ellas.

Los alumnos de la ETSID actuaban en el rol de diseñadores de las empresas y debían crear la filosofía y la imagen de marca, los logotipos y los envases para cada uno de los cuatro productos desarrollados por los alumnos de la ETSIAMN. Los alumnos de la ETSIAMN actuaban por tanto como clientes de los alumnos de la ETSID.

El desarrollo de esta fase del proyecto se realizó en base a sesiones específicas previas independientes para los alumnos de la ETSIAMN y ETSID; sesiones conjuntas para las dos titulaciones; sesiones de trabajo de los equipos interdisciplinares y por último se desarrolló partes del trabajo de forma independiente entre los miembros de los subgrupos.

\section{1) Sesiones con los alumnos de la ETSIAMN}

El Design Thinking (DT), se define como la utilización de técnicas de exploración de un problema, buscando soluciones diversas para un posterior prototipado y testeo. De forma general el proceso básico del DT consta de cuatro pasos elementales (figura 1).

(cc) EY-NC-ND 2018, Universitat Politècnica de València

Congreso IN-RED (2018) 


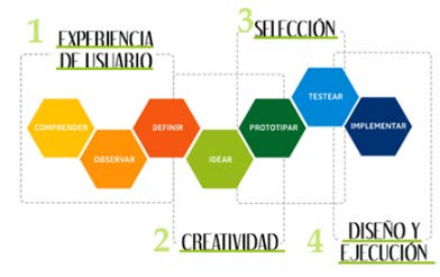

Fig.1. Esquema del proceso básico del DT (fuente: Institute Of Design at Stanfor, 2016)

La primera etapa constituye la experiencia, que incluye la comprensión y la observación del usuario, para poder definir el problema real que presenta. Este paso va seguido de la aplicación de la creatividad al proceso de como que se generan ideas. A continuación, mediante un proceso de selección de la mejor idea, se pasa al prototipado para obtener una propuesta que se adapte a los requisitos establecidos. Por último, se lleva a cabo la implementación y ejecución del prototipo.

En base a lo anteriormente mencionado se realizaron diversas sesiones de trabajo para implementar las distintas fases del DT en la elaboración de un producto alimentario, utilizándose algunas de la herramientas definidas en la guía elaborada en el marco del Proyecto Europeo Erasmus+ FoodLab, en el que han participado varios de los profesores involucrados en esta innovación docente (FoodLab, 2018) (figuras 2 y 3).

Posteriormente, también se realizaron varias sesiones de seguimiento sobre la elaboración del modelo de negocio de los productos desarrollados a través del Business Canvas.
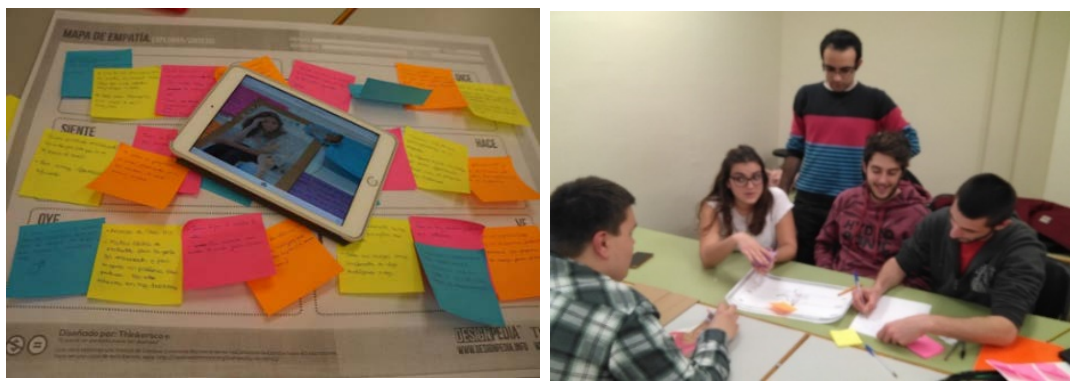

Fig. 2. Sesión de trabajo de los alumnos utilizando la herramienta EMPATIZAR
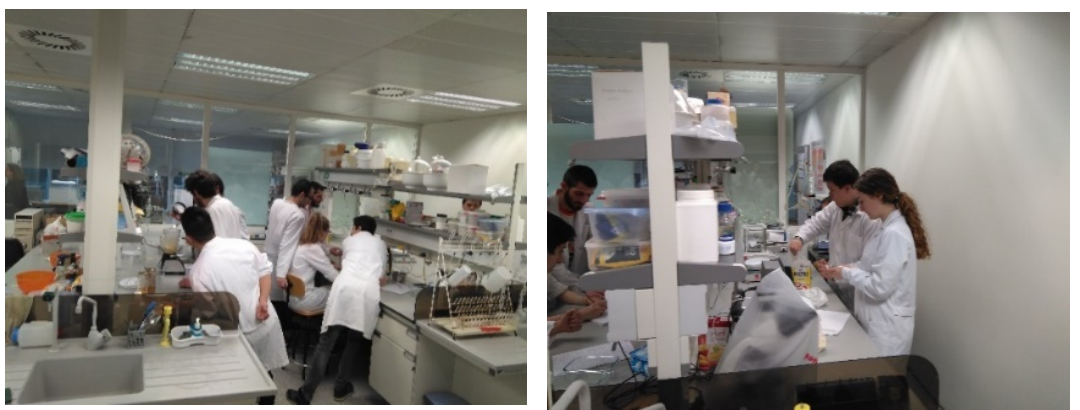

Fig. 3. Sesiones de PROTOTIPADO.

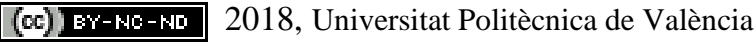

Congreso In-Red (2018) 


\section{2) Sesiones con los alumnos de la ETSID.}

Para los alumnos de la asignatura de Color y diseño del Grado en Ingenieria del Diseño y Desarrollo de Productos de la ETSID, la participación en este proyecto era la aplicación práctica de los conocimientos teóricos estudiados al inicio de la asignatura (Febrero y Marzo 2017), sobre el Diseño de productos y la fuerza de la percepción cromática para la determinación de una idea o concepto. Durante dos sesiones se preparó a los alumnos para realizar un cuestionario claro de los aspectos claves a tratar con sus "clientes" para poder definir de manera colectiva con ellos, la filosofía e imagen final del producto alimentimentario. Además, durante el proceso de creación de las propuestas los subgrupos se reunieron con el profesorado con el fin de recibir asesoramiento y apoyo.

\section{3) Primera sesión conjunta para las dos titulaciones .}

Una vez ideado el producto por parte de los alumnos de la ETSIAMN, se realizó una primera toma de contacto en una sesión conjunta, para que estos presentansen el producto a los alumnos de la ETSID (figura 4) y así, estos últimos, pudiesen proceder a definir la filosofía y la imagen de marca, incluyendo el logotipo y el envasado. En esta misma sesión y tras las presentaciones, los equipos de la ETSID eligieron en que producto alimenticio iban a trabajar. Cada tres grupos de alumnos de la ETSID trabajaría con un grupo de alumnos de la ETSIAMN para desarrollar el concepto completo del nuevo producto. Por tanto, cada producto alimentario tendría tres propuestas de diseño de dónde se elegiría una para pasar a la fase de concurso de Ecotrophelia.

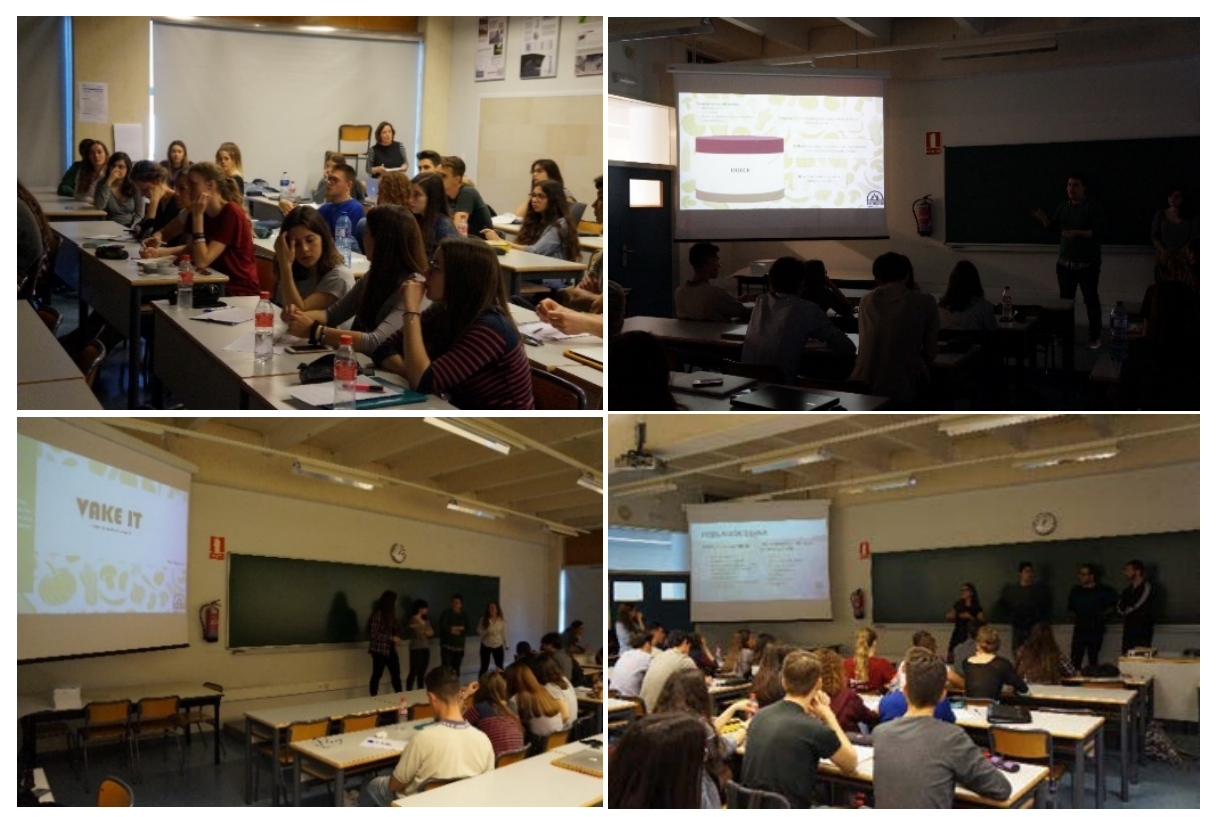

Fig. 4. Primera sesión conjunta ETSIAMN y ETSID. 


\section{4) Sesiones de trabajo de los equipos interdisciplinares.}

Durante estas sesiones de trabajo conjunto que tuvieron lugar durante los meses de Marzo y Abril 2017, los subequipos interdisciplinares de ambas escuelas se trasmitierón información básica sobre los productos alimenticios propuestos, trabajarón sobre la definición del usuario y el mercado de destino, sobre las características de conservación y la percepción del producto en el comercio final, entre otros aspectos esenciales para el desarrollo de la filosofía e imagen de la marca y de la empresa, con el objetivo común de presentar un resultado final coherente, completo y atractivo. La organización y comunicación de estas sesiones corrió a cargo de los equipos interdisciplinares, sin necesidad de la presencia de un profesor.

\section{5) Segunda sesión conjunta para las dos titulaciones.}

En la segunda sesión conjunta entre los alumnos de las dos escuelas involucradas en el proyecto, los estudiantes de la ETSID presentaron los envases, logotipos e imagen de marca para los cuatro productos alimentarios ideados por los alumnos de la ETSIAMN (figura 5). El objetivo de la sesión era la elección por parte de los equipos de la ETSIAMN, una de las tres propuestas presentadas por los subgrupos de la ETSID y seguir con ella la siguiente fase del proyecto que consistión en la presentación en ECOTROPHELIA 2017.
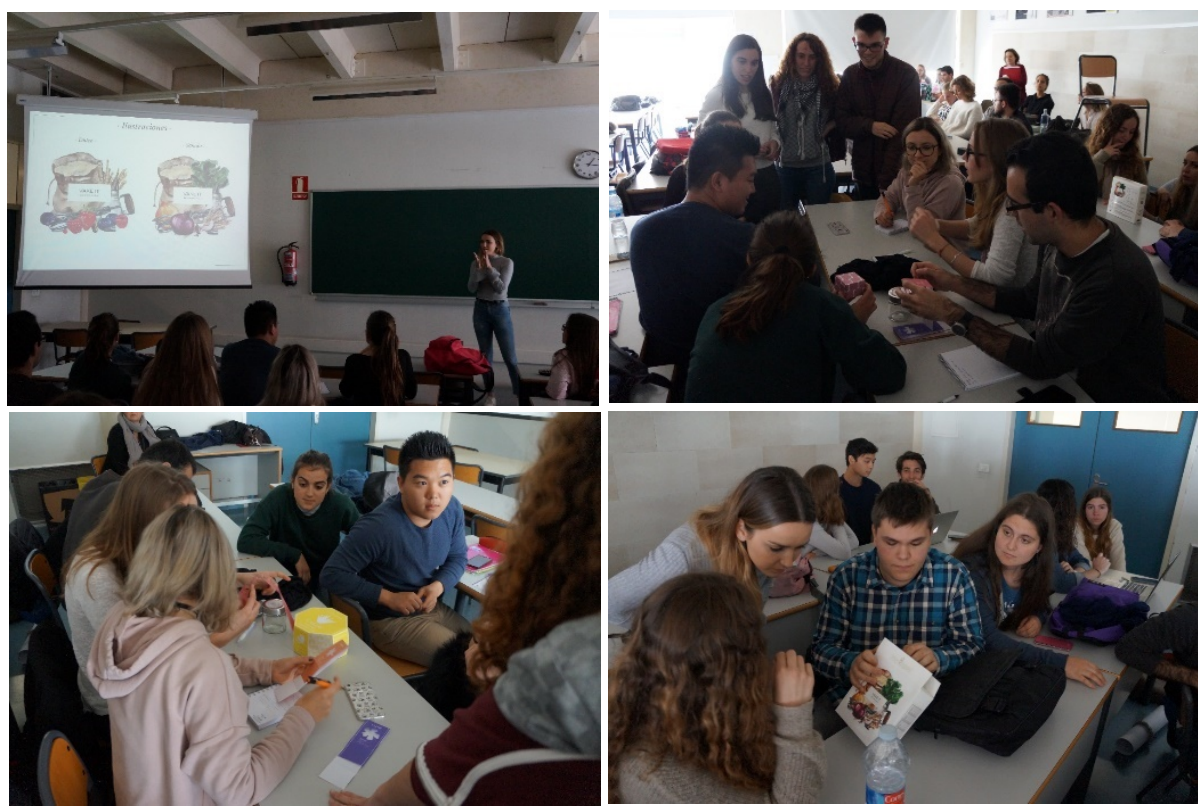

Fig. 5. Segunda sesión conjunta ETSIAMN y ETSID.

6) Presentación de los productos resultantes (producto+filosofía e imagen de marca+envase) al concurso ECOTROPHELIA 2017 (Barcelona, 22 mayo 2017) (figura 6). 


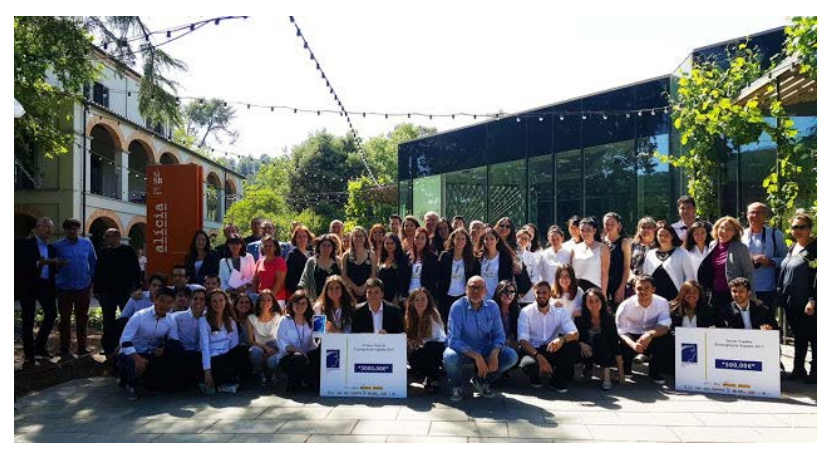

Fig. 6: participantes al certamen ECOTROPHELIA 2017.

En la figura 7 se muestra el análisis DAFO realizado por parte de los participantes en el proyecto respecto al desarrollo de este.

\begin{tabular}{|c|c|}
\hline DEBILIDADES & FORTALEZAS \\
\hline $\begin{array}{ll}\text { - } & \text { Organización compleja } \\
\text { de las sesiones por el } \\
\text { número de alumnos. } \\
\text { - } \\
\text { Dificultades de } \\
\text { comunicación entre } \\
\text { alumnos procedentes de } \\
\text { Escuelas diferentes. } \\
\text { - Tiempo y plazos cortos. }\end{array}$ & $\begin{array}{ll}\text { - } & \text { Multidisciplinaridad } \\
\text { - } & \text { Complementariedad } \\
\text { - } & \text { Trabajo en equipo } \\
\text { - } & \text { Experiencia real y activa } \\
\text { - } & \text { Motivación } \\
\text { - } & \text { Objetivos cognitivos de los } \\
& \text { niveles más altos }\end{array}$ \\
\hline AMENAZAS & OPORTUNIDADES \\
\hline $\begin{array}{ll}\text { - } & \text { Coordinación de cronograma } \\
\text { - } & \text { Organización entre escuelas }\end{array}$ & $\begin{array}{ll}\text { - } & \text { Creación de Start Up } \\
\text { - } & \text { Participación en certámenes } \\
\text { - } & \text { Creación de redes de trabajo para } \\
& \text { noveles } \\
\text { - } & \text { Creación de equipos } \\
& \text { multidisciplinares entre escuelas } \\
\text { - } & \text { Emprededurismo multidisciplinar }\end{array}$ \\
\hline
\end{tabular}

Fig. 7: Analisis DAFO del PIME.

\subsection{Definir las competencias transversales trabajadas en el proyecto.}

Para establecer las competencias transversales trabajadas en el presente proyecto se procedió a realizar encuestas a los diferentes colectivos de alumnos utilizados como grupos de control y definidos en el apartado 3.1.

(cc) EY-NC-ND 2018, Universitat Politècnica de València 


\section{Resultados}

\subsection{La implementación del aprendizaje basado en un proyecto real entre grupos interdisciplinares de diferentes titulaciones.}

Los resutlados obtenidos fueron la presentación de cuatro productos con el desarrollo completo incluyendo imagen y plan de negocio, al certamen Ecotrofelia España 2017.

- SEABITES es una gominola funcional a base de microalgas para tomar durante una actividad física aeróbica intensa (figura 8).

- LENTEYA un plato de lentejas liofilizadas que solamente se reconstituye con agua y contenidas en un envase autocalentable, lo cual resulta ideal para personas sin tiempo para cocinar por cuestiones de trabajo, o sin recursos para cocinar como los excursionistas (figura 9).

- VAKE IT es un preparado para tarta que no incluye ningún ingrediente de origen animal el cual solo necesita refrigeración para su consumo. El preparado incluye todos los ingredientes que el consumidor necesita para realizar este postre, a excepción del agua, necesaria para elaborar el relleno de la tarta, y en algunas ocasiones de la grasa, necesaria para compactar la base. Dentro de VAKE IT se presentan dos modelos: uno de tarta salada y otro modelo de tarta dulce (figura $10)$.

- CALOE es una gama de mousses a base de bebida y yogur de soja con la peculiaridad de que incluye entre sus ingredientes jugo de aloe vera y flores (figura 11).

Sobre un total de quince productos presentados en la primera fase del concurso ECOTROPHELIA España en 2017 siete parasaron a la fase final y estos tres fueron productos presentados por la UPV (LenteYa, Vake It y Caloe).

el producto

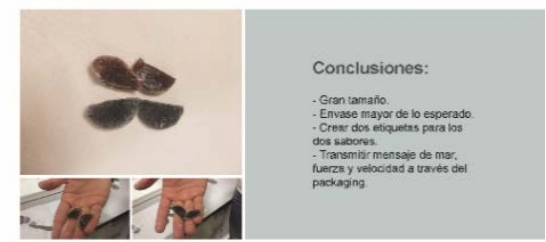

diseño de envase

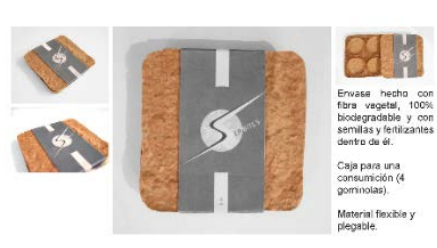

Fig.

8: 


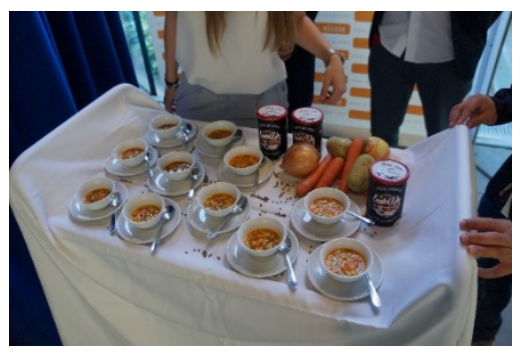

Fig. 9. LenteYa

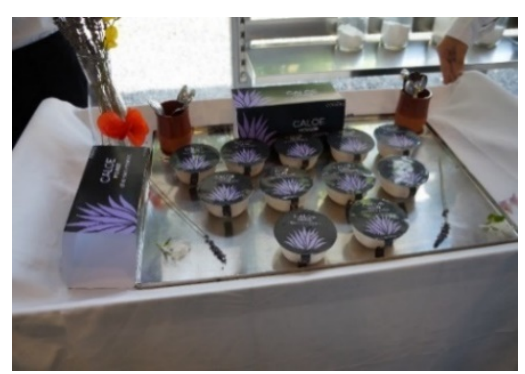

Fig. 11. Caloe

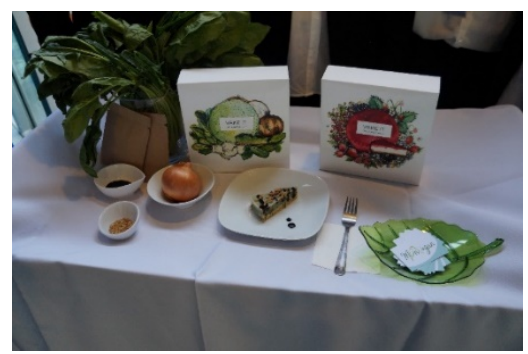

Fig. 10. VakeIt.

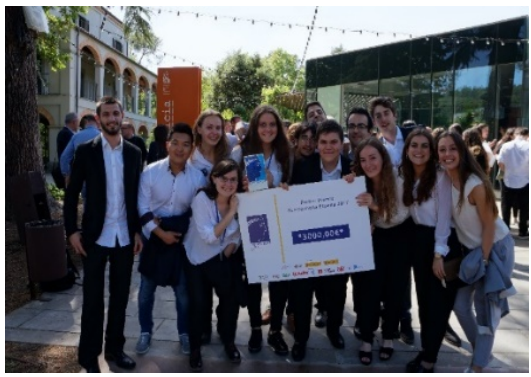

Fig. 12. Vake It ganador del certamen

En la fase final del concurso Vake It quedo ganador y representará a España en ECOTROPHELIA Europa que se desarrollará en Londres el 21 y 22 noviembre 2017 (figura 12).

Para evaluar la metodología seguida y las herramientas utilizadas en el caso de los alumnos de la ETSID se utilizó una escala a tres niveles: insatisfactorio (puntuación 1, 2, 3), satisfactorio (puntuación 4) y excelente (puntuación 5). Los resultados obtenidos se muestran en la figuras 14 . Para evaluar la metodología seguida y las herramientas utilizadas, en el caso de los alumnos de generación espontanea de la ETSIAMN, se utilizó una escala de 1 a 5 donde 1 indica un fuerte desacuerdo y 5 indica un fuerte acuerdo. Los resultados se muestranen la figura 15 . 


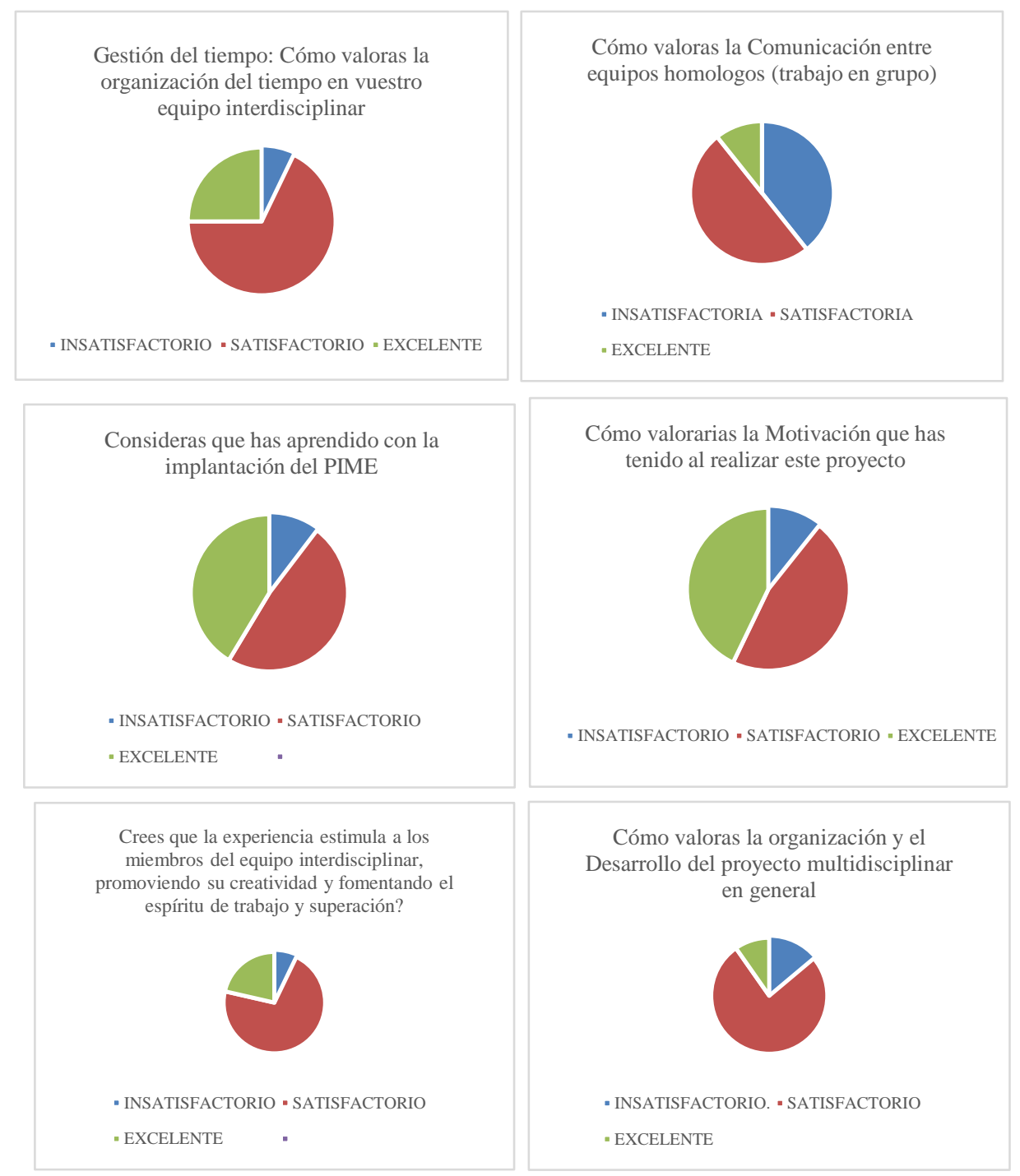

Fig. 14 Resultados de las encuestas realizadas a los alumnos de la ETSID.

\begin{tabular}{|l|l|}
$\begin{array}{l}\text { Cómo valoras los resultados del } \\
\text { proyecto? }\end{array}$ & $\begin{array}{l}\text { Cómo valoras la Transmisión de } \\
\text { información entre los equipos }\end{array}$ \\
- INSATISFACTORIO - SATISFACTORIO & - INSATISFACTORIO - SATISFACTORIO \\
"EXCELENTE EXELENTE
\end{tabular}

(cc)) EY-Nc-ND 2018, Universitat Politècnica de València

Congreso In-Red (2018) 
ECOTROPHELIA 2017: Una oportunidad para el enfoque interdisciplinar en el aprendizaje basado en proyectos.

Nueva Metodología:El proyecto te ha

servido para integrar los conocimientos adquiridos en la asignatura

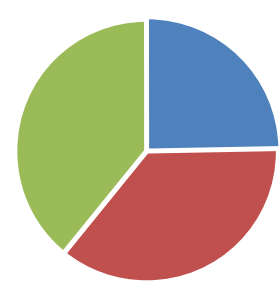

INSATISFACTORIO -SATISFACTORIO - EXCELENTE.

Fig. 14 (bis): resultados de las encuestas realizadas a los alumnos de la ETSID.

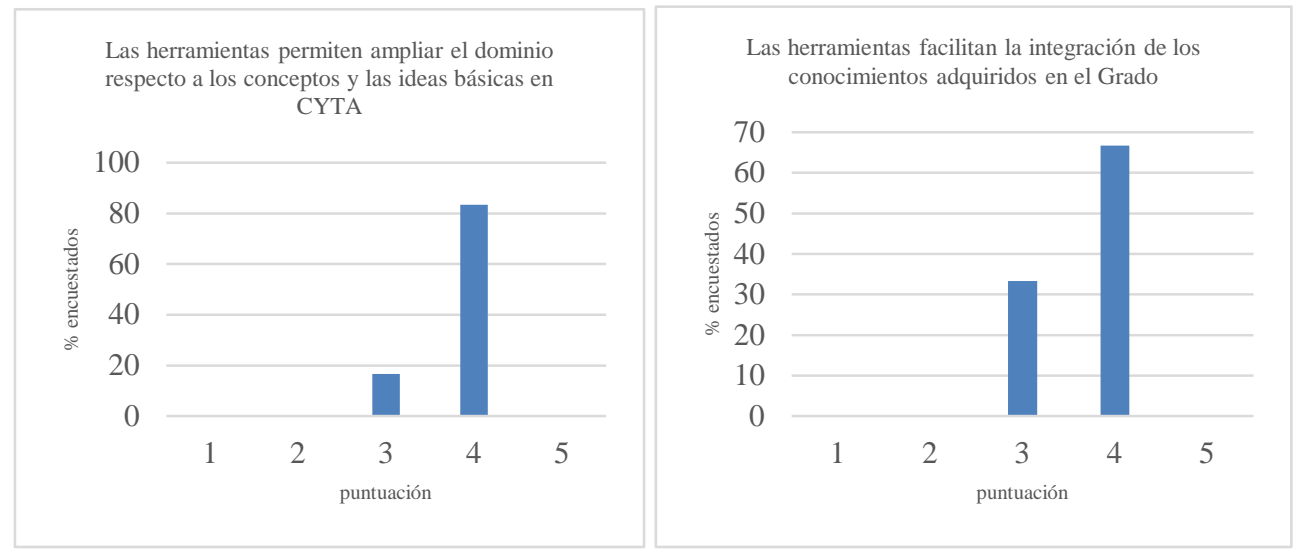

Fig. 15. resultados de las encuestas realizadas a los alumnos de la ETSIAMN

(c)) EY-NC-ND 2018, Universitat Politècnica de València 


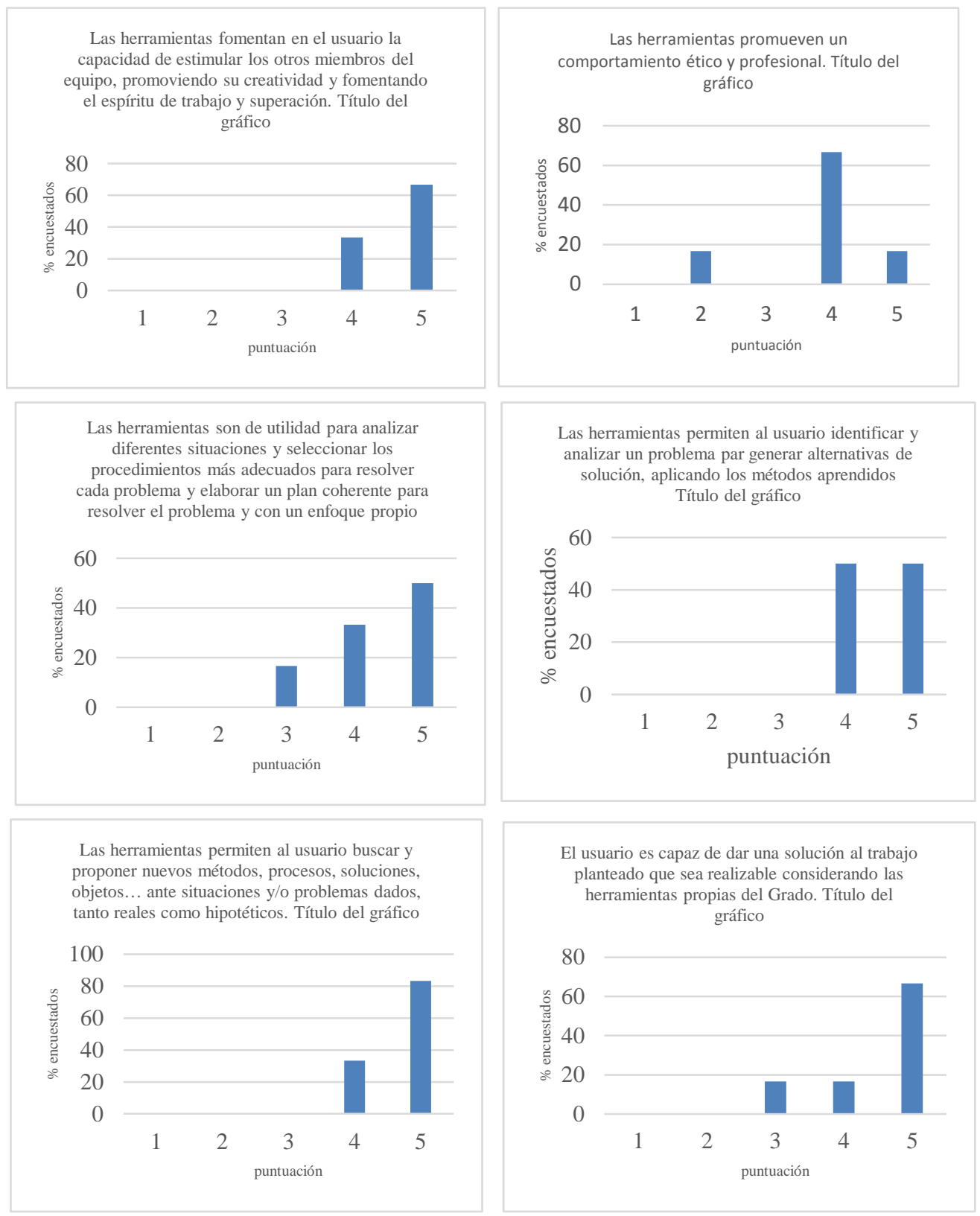

Fig. 15 (bis). resultados de las encuestas realizadas a los alumnos de la ETSIAMN.

(c)) EY-NG-ND 2018, Universitat Politècnica de València 
ECOTROPHELIA 2017: Una oportunidad para el enfoque interdisciplinar en el aprendizaje basado en proyectos.

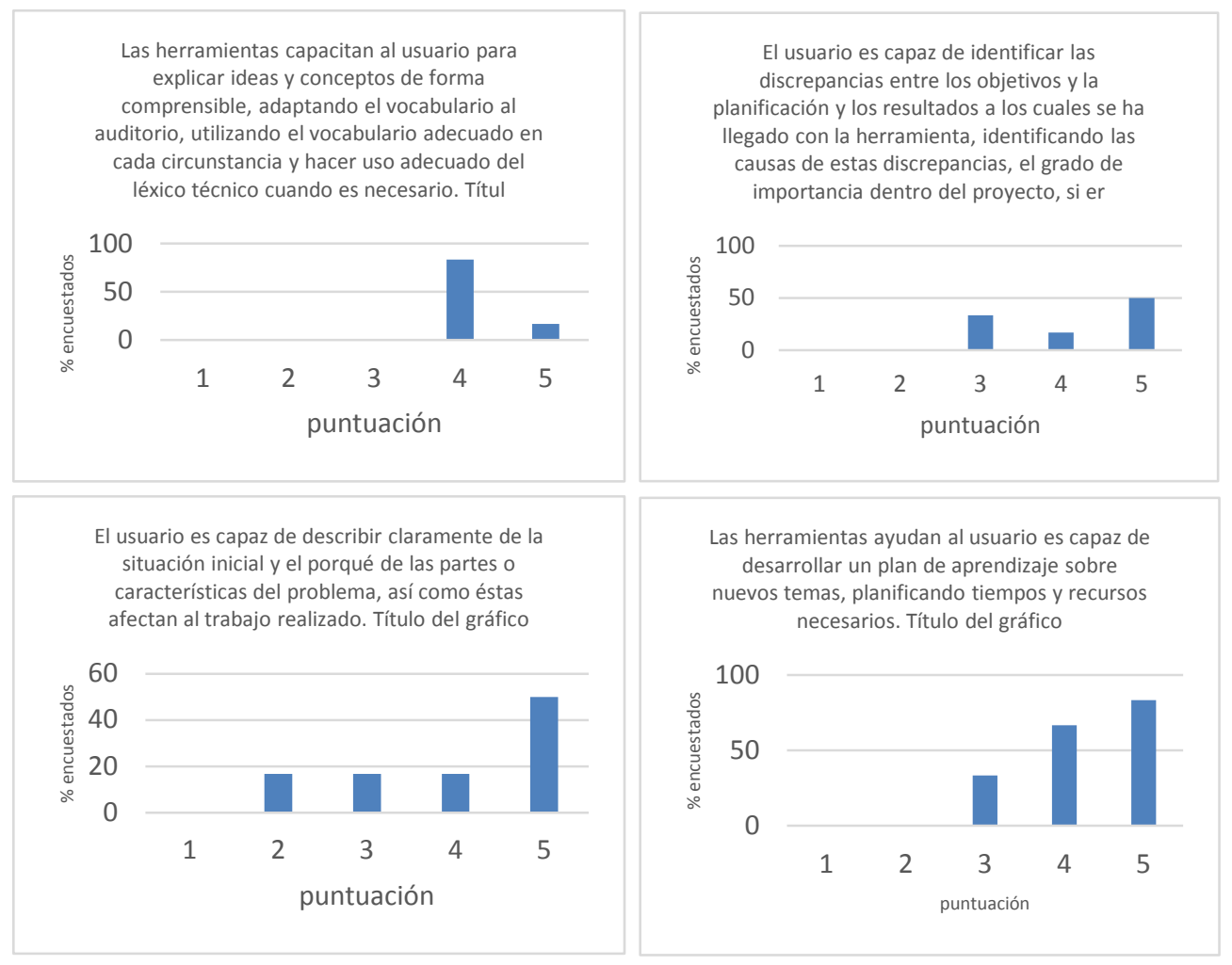

Fig. 15 (tris). resultados de las encuestas realizadas a los alumnos de la ETSIAMN.

\subsection{Competencias transversales trabajadas durante el proyecto.}

En base a los resultados expuestos en el apartado anterior se puede concluir que las competencias transversales fundamentales que se han trabajado en paralelo al desarrollo de este tipo de proyecto son todas las recogidas por la UPV:

C1: comprensión e integración

C2: aplicación y pensamiento práctico

C3: análisis y resolución de problemas

C4: innovación, creatividad y emprendimiento

C5: diseño y proyecto

C6: trabajo en equipo y liderazco

C7: responsabilidad ética, medioambiental y profesional

C8: comunición efectiva

C9: pensamiento critíco

(cc) EY-Nc-ND 2018, Universitat Politècnica de València

Congreso IN-RED (2018) 
C10: conocimiento de problemas contemporáneos

C11: aprendizaje permanente

C12: planificación y gestión del tiempo

C13: instrumental especifico

Queremos aclarar que no todas las competencias expuestas, fueron trabajadas con la misma profundidad por los dos colectivos de alumnos, debido a que cada colectivo tubo roles diferentes dentro del desarrollo del proyecto, lo cual permitió la complementariedad de los procesos, pero con ello también, el trabajo más concreto sobre diferentes competencias transversales .

\subsection{Metodología y técnicas utilizadas para el tratamiento y análisis de los datos.}

Se han utilizado tres tipos de encuestas, en función de la finalidad perseguida:

- Encuesta para la valoración de las herramientas para la creación del plan de negocio (ETSIAMN)

- Encuesta para la valoración de la metodología seguida en el desarrollo del proyecto desde el punto de vista del aprendizaje interdisciplinar ybasado en proyectos centrándonos en las competencias transversales (ETSIAMN y ETSID)

Además, en la fase 3 del proyecto, se ha contado con una evaluación externa, al estar compuesto el jurado de ECOTROPHELIA por profesionales de la Industria Alimentaria. El jurado del certamen estuvó presidido por Mauricio García de Quevedo, Director General de FIAB, y Toni Massanés, Director General de Fundación Alicia, además de por grandes expertos de la $\mathrm{I}+\mathrm{D}+\mathrm{i}$ del sector en España, desde la Administración pasando por las empresas del sector y centros de investigación de renombre nacional.

\section{Conclusiones}

Tras la experiencia y el análisis de los resultados, podemos concluir que el proyecto de innovación docente permitió al alumno llegar a alcanzar el aprendizaje completo, viniendo a reafirmar la existencia de una conexión muy intima entre conocimiento y práctica. Los estudiantes pueden cambiar y desarrollarse, no sólo por la adquisición de conocimientos, sino por el querer resolver situaciones que les resultan interesantes, atractivas o importantes, trabajando objetivos cognitivos de los niveles más altos.

A su vez, el Aprendizaje Basado en Proyectos (ABP) es una metodología de gran interés en el área de los estudios técnicos e ingenieriles. Si su aplicación trasciende a varias asignaturas de titulaciones diferentes, consideramos que sus beneficios se ven incrementados por la transversalidad, multidisciplinariedad y la riqueza del desarrollo de un proyecto completo y coherente. Sin embargo, queremos remarcar que la mayor dificultad en la actualidad para la implantación de este innovación supone todavía, la coordinación entre alumnos de dos colectivos diferentes a la hora de programar el proyecto y desarrollarlo. 
ECOTROPHELIA 2017: Una oportunidad para el enfoque interdisciplinar en el aprendizaje basado en proyectos.

En definitiva consideramos tras esta experiencia, que el logro de la excelencia universitaria pasa sin duda, por el enfoque interdisciplinar de la docencia como respuesta a la demanda futura de profesionales. Se nos exige que preparemos profesionales con competencias técnicas, pero sobretodo profesionales preparados para aprender a aprender y aprender a trabajar juntos y hemos constatado que podrán educarse de este modo siempre y cuando, seamos capaces de romper los límites entre disciplinas.

\section{Referencias}

ARIAS OLIVA,M (2003) El espacio Europea de Educación Superior: una oportunidad de desarrollo multidisciplinar a través del aprendizaje y la tecnología. Universitat Rovira i Virgili [en línea] Disponible en web: https://repositorio.uam.es/bitstream/handle/10486/680281/EM_15_3.pdf?sequence=1 [ref. 20 de Marzo de 2018].

BAIN,K (2005) Lo que hacen los mejores profesores universitarios, Publicacions de la Universitat de València, pp 229

BROWN, G \& ATKINS,M (1998), Effective Teaching in higher education .Methuen\&Co.Ltd, pp. 50-90.

DELORS, J. (1996): La Educación Encierra un Tesoro. Informe a la UNESCO de la Comisión Internacional para la educación del Siglo XXI. UNESCO

EDVINSSON,L \& MALONE,M.S (1997) Intellectual Capital, Harper Collins Publisher INC., New York.

FOODLAB, EUROPEAN FOOD INCUBATOR (2018). ((https://foodlab-eu.com/) [ Consulta: 20 marzo de 2018].

FULLAN,M (2002) Los nuevos significados del cambio en la educación, Ed.Octaedro.

GONZALEZ-AURIGNAC, E \& SAIZ MAULEON, B (2013) Innovación docente en la metodología aplicada a la asignatura Taller de Diseño I Jornadas de Innovación Docente ICE 2013, UPV.

INSTITUTE OF DESIGN AT STANFOR (2016). An introduction to Design Thiking process guide. [online], Avaliable at: http://dschool.stanford.edu/. [Consulta: 20 marzo de 2018].

LAVE, J \& WENGER E (1991) Situated Learning. Legitimate peripheral participation, Cambridge: University of Cambridge Press, pp 138 . 